Bawden, F. C. (1955). J. gen. Microbiol. 12, 362-366

\title{
The Classification of Viruses
}

\author{
By F. C. BAWDEN \\ Rothamsted Experimental Station, Harpenden, Hertfordshire
}

Before considering the criteria to use for classifying plant viruses, it is first necessary to consider for what purpose we are classifying them. This fact applies to whatever is being classified and might seem too self-evident to need saying, except that taxonomists often give the impression that there is only one classification possible in biology; this is one based on what are usually termed 'natural' relationships, by which seems to be meant that the groupings reflect current views on evolution. To speak of classifying plants, for example, almost invariably brings to mind only the classification developed by academic botanists, in which plants are first analysed into the basic units of species and then grouped for phylogenetic reasons into genera, families and orders. There are, though, many other possibilities, and few could be less useful than the botanist's to those who are engaged in the cultivation of plants. The main concern of the farmer or gardener is not whether a plant is graminaceous or cruciferous, but whether it is a useful plant for him to grow or a pernicious weed. To the botanist, couch grass may be a near relative of wheat, and charlock of turnips, but to the farmer one of each pair means a profit and the other a loss, two categories that, to his mind, could not be more unrelated. The farmer needs plants classified in categories that show their agricultural value, the way in which they respond to climate, soil type and manuring, their habits of growth, methods of propagation, and whether they are annuals, biennials or perennials. Such classifications also have the virtue of being factual and permanent.

Similarly, viruses can be classified for the farmer or field pathologist quite objectively, by using such criteria as the susceptibility of specific plants to infection, the kind of symptom evoked, the method of spread and, if spread by arthropods, by the species of the vector. To expect such criteria to reflect any phylogenetic relationships between the viruses placed in any one category, however, would be as unrealistic as to expect the farmer's classification of plants to coincide with the botanist's. I imagine that to-day we are less concerned with utilitarian groupings of viruses than with classifications that will be acceptable to the taxonomists of microbiology. Let it be said at once that such classifications are not only more difficult to make, but they must be more equivocal and they also lack any agreed criteria. Indeed, it is even doubtful whether viruses are suitable objects for attempting to arrange in the kinds of groups that are used to classify organisms; the determination of such groups depends on sexual reproduction and phylogeny, criteria that could hardly be less appropriate for classifying clones about whose origin we have not the slightest idea. Other criteria must therefore be sought. 
Classification of organisms consists of two things: one, the analysis of individuals into basic units and, two, the synthesis of these units into like groups. The analysis, I think, presents no great problems with plant viruses, but the synthesis does. Whether or not our units should be called species, is something I shall not pursue to-day. I shall simply follow Mayr and call the unit a 'collective species', by which I shall mean a collection of clones that have many features in common, but differ in some, the most frequent difference so far detected (probably only because it is the easiest to detect) being in pathogenicity. Later work may show that they are true species, in the sense now customarily used for organisms, meaning that the clones of one 'collective species' will inter-breed, but will not cross with clones of another 'collective species'. Recent work with bacteriophages suggests that, when related clones multiply in one cell, they can exchange genetic characters, and techniques may be developed in the future to test whether or not clones of plant viruses can also do so. If they can, we shall have an objective and generally acceptable test for deciding inclusion in a species, but that time still seems far off. Until then we must use some other criterion, preferably one that is also objective, even though we are doubtful of the relationship it discloses. The criterion that seems to me by far the most valuable is the sharing of common antigens. All the viruses so far studied that have been found to be related serologically have also been found to resemble one another in many other intrinsic properties. They have similar sizes and shapes, similar stabilities in vitro and similar gross chemical constitutions.

Our first step in classification should be to select specific clones as types of named 'collective species', such as tobacco mosaic, potato $X$, potato $Y$, and so on, and antisera should be prepared against each type. Then, by testing individual virus clones against the type antisera, the clones can be allotted to 'collective species' according to the antiserum with which they react. This would be an immense step forward; it would get rid of many synonyms that now plague the literature, and it would show which of the 'new' viruses that are continually being recorded are really new and which are related to already named types. Indeed, it might so decrease the numbers of 'recognized' viruses, that the need for further classification might seem less urgent than it now does.

Unfortunately, serological techniques, although readily applicable to many plant viruses, are not applicable to all. This does not necessarily mean that some plant viruses are not antigenic; more likely it means only that they are too readily inactivated, or occur in too small amounts, to be studied by current serological techniques. No doubt refinements of these techniques will ultimately allow many viruses that are now not amenable to serological study to be handled successfully. There is, though, no need to wait for these refinements before attempting to allot many of these viruses to provisional 'collective species'. Another criterion can be applied; it is to test whether clones can interfere with each other's multiplication in susceptible plants, a feature that seems to be closely tied to serological relationship. Viruses that are serologically unrelated to one another usually fail to depress the multiplication of 
each other, and plants infected simultaneously with two such viruses usually show more severe diseases, and often lesions of quite a different type, from those produced by either virus alone. By contrast, if two serologically related viruses are inoculated simultaneously to a plant, they depress each other's multiplication, and the resulting symptoms are usually intermediate between those caused by either alone. Also, a plant already fully invaded by one virus, resists invasion by a second that is serologically related, but not by one that is serologically unrelated. The mechanism underlying the resistance is unknown, but this need not concern us to-day, when the only point of importance is that it is in some way correlated with the antigenic structure of the virus particles and so can be used as a supplementary test for allocating virus isolates to 'collective species'.

It is too much to expect that the plant protection test will always give such unequivocal results as do serological tests, but that it will sometimes fail is no reason for not using it when it succeeds. It will probably give equivocal or false results when the two viruses being tested are only remotely related serologically, for, with only one exception as yet, viruses that share many antigens have protected plants against one another unequivocally, whereas the protection has become increasingly feeble as the number of common antigens decreases. This need not be regarded simply as a deficiency of the test; if carefully used it may be an added value, for it may indicate degrees of relationship between clones. Certainly serological tests seem able to do this. Plant viruses are multiple antigens, and individual clones of a 'collective species' often contain different numbers of common antigens. If it is reasonable to assume that all serologically related clones derive from one original source, those that share many antigens, or that completely protect plants against one another, are also reasonably regarded as more recently derived from a common stock, than those that share only a few antigens or only slightly interfere with each other's ability to multiply in infected plants. In this connexion it is perhaps worth comment that clones of tobacco mosaic virus that are closely related serologically seem also to have similar amino acid constitutions, isoelectric points, electrophoretic mobilities and resistance to inactivation by ultraviolet radiation, whereas those that contain only a few common antigens often differ considerably in such properties. As those with few common antigens also often have widely differing host ranges, the inference is that they have been evolving in isolation from one another.

There is, then, the possibility that within 'collective species' clones might be arranged in some sequence that reflects phylogeny, but beyond this it seems impossible to try to use evolution as a basis for grouping. All the plant viruses that have so far been purified have been found to be nucleoproteins, and their origins are wholly obscure. Each 'collective species' seems to have an equal chance of deriving from a higher plant, a micro-organism, an insect or any other kind of organism, for nucleoproteins are components of all living cells. The fact that we cannot group our 'collective species' by inferred phylogeny is one of the reasons that makes me strongly oppose the use of Linnaean binomial names for plant viruses. These names not only demand 
identification at the species level, which $I$ hope $I$ have shown can be done, but the arrangement of species into genera, and the word genus to a modern taxonomist suggests a group of phylogenetically related species that is clearly separated from other genera.

The 'collective species' can, of course, be grouped on criteria other than phylogeny, but then there must be no pretence that the categories do what genera and families do for organisms. The obvious first choice as a criterion is morphology, and present evidence from electron microscopy suggests that three categories, corresponding roughly to bacilli, spirochaetes and cocci, could be made depending on whether the particles are seemingly rigid rods, flexible filaments or spheres. Whether any significance should be given to size, is anybody's guess, for the chemical constitution of no large virus is yet known. If large size should be found to reflect greater chemical complexity, then we shall have a further criterion for grouping according to constitution. At present, however, all plant viruses whose constitution has been determined contain only ribose nucleic acid and protein, and the main difference between 'collective species' is the relative proportions of the two components. It may be taxonomically significant, and a prop to using shape as a criterion for grouping, that, of the viruses so far analysed, those with elongated particles all have the same ratio of nucleic acid to protein, whereas those with spherical particles all have three or more times as much nucleic acid. Although chemistry is not now particularly helpful taxonomically, it can be expected to become so in the future, for if, as is likely, the grouping of viruses is to be achieved on details of morphology, these details are most likely to be shown by the techniques of protein chemistry.

Many plant viruses are not now amenable to study by the electron microscope and so they cannot be grouped according to their gross shapes. Some of these cause similar diseases (the 'yellows' type) in many different hosts, and they are transmitted similarly by leaf-hoppers. Faute de mieux these might temporarily be grouped because of such behaviour, but the groupings should be abandoned as soon as such extrinsic characters can be replaced by intrinsic ones. There are too many examples already known of individual clones of one 'collective species' differing widely in pathogenicity and in ability to be transmitted by a given insect, to place any taxonomic reliance on such characters. The main value of pathogenicity and transmission by a vector is not for indicating relationships, but for showing differences between clones of one 'collective species' that otherwise would seem identical.

\section{DISCUSSION}

\section{By A. Felix, Lister Institute, London}

My remarks are provoked by Dr Andrewes's and Dr Bawden's relapses into the bad habit of using observations on bacteriophages in order to explain some of the difficulties with animal and plant viruses. In support of my own views 
I quote from Burnet's recent article in the Med. J. Aust. (28 November 1953, p. 809): 'However, I am one of those who in the last year or two have come to believe that the viruses responsible for human and animal disease are not so close to the bacterial viruses as we once thought.' Dr Bawden has been too optimistic about serology and too pessimistic about chemistry in their respective roles as tools in virology. So far as bacteriophages are concerned 'protection tests' or 'interference tests' certainly are no proof of identity of, or even similarity in, antigenic composition. 